\title{
A FIELD OF COHOMOLOGICAL DIMENSION 1 WHICH IS NOT $C_{1}$
}

\author{
BY JAMES AX \\ Communicated by A. Rosenberg, April 5, 1965
}

Let $k$ be a field of characteristic $q$ (=prime or 0 ) and let $r$ be a nonnegative integer. Then $k$ is said to be $C_{r}$ if and only if every (homogeneous) form of degree $d$ in $n$ variables over $k$ has a nontrivial zero over $k$ if $n>d^{r}$. In Serre [4, Chapitre II, Corollaire to Proposition 8] the following result is obtained: If $k$ is $C_{1}$ then $\operatorname{dim}(k) \leqq 1$ and $\left[k: k^{q}\right]$ $=1$ or $q$. Here $\operatorname{dim}(k)$ is defined cohomologically. Serre then remarks:

"On ignore si la réciproque du corollaire précédente est vrai-c'est peu probable."

Actually, the problem of the relation of cohomological dimension $r$ and $C_{r}$ had been previously raised in Serre [3]. We exhibit below a field $R$ of characteristic zero of dimension 1 which is not $C_{1}$. This implies, for all $r \geqq 1$, the existence of fields of dimension $r$ which are not $C_{r}$. But the situation is worse than that: $R$ is quasi-finite in the sense of Serre [2, Chapitre XIII, §2], and for all $r, R$ is not $C_{r}$. The interest in these considerations stemmed from a possible relation with Artin's conjecture which states: If $k$ is a totally imaginary number field or a $p$-adic field, then $k$ is $C_{2}$. Indeed, for such fields $k, \operatorname{dim}(k)=2$ as is proved in Serre [4, Chapitre II, Corollaire to Proposition 12, Proposition 13].

We now define $R$. Let $F$ be an algebraically closed field of characteristic zero. If $K$ is a field, then $K((t))$ denotes the field of formal power series in $t$ over $K$. Let $F_{2}=F\left(\left(t_{2}\right)\right)\left(t_{2}^{1 / n}: 2 \nmid n\right)$. If $p$ is a prime greater than 2 and $q$ is the largest prime less than $p$, we recursively define $F_{p}=F_{q}\left(\left(t_{p}\right)\right)\left(t_{p}^{1 / n}: p \nmid n\right)$. Finally we set $R=\operatorname{inj} \lim _{p} F_{p}$.

TheOREM. $R$ is quasi-finite, but $R$ is not $C_{r}$ for any $r$.

The proof will appear in Ax [1].

\section{REFERENCES}

1. J. Ax, Proof of some conjectures on cohomological dimension, Proc. Amer. Math. Soc. (to appear).

2. J-P. Serre, Corps locaux, Hermann, Paris, 1962.

3. Cohomologie galoisienne des groupes algêbriques lineaires, Colloque de Bruxelles, 1962, pp. 53-67.

4. - Cohomologie Galoisienne, Mimeographed notes, College de France, 1963 (also as Lecture Notes in Mathematics, No. 5, Springer, Berlin, 1964).

CORNELL UNIVERSITY 